\title{
Research status and applications of nature-inspired algorithms for agri-food production
}

\author{
Yanbo Huang \\ (USDA-ARS Crop Production Systems Research Unit, Stoneville, MS 38776, USA)
}

\begin{abstract}
Nature-inspired algorithms have been developed with biological mimicking. Machine learning algorithms from artificial neurons and artificial neural networks have been developed to mimic the human brain with synthetic neurons. This research can be traced back to the 1940s and has been expanded to agri-food problem solving in the last three decades. Now, the research and applications have entered the stage of deep learning with more layers and neurons that have complex connections to extract deep features of the target. In this paper, the developments of artificial neural networks and deep learning algorithms are presented and discussed in conjunction with their biological connections for agri-food applications. The related independent studies previously conducted by the author are summarized with the newly conducted being presented. At the same time, the algorithms motivated by recent bionics studies are compared and discussed for their potentials for agri-food production.
\end{abstract}

Keywords: nature-inspired algorithm, agri-food production, machine learning, deep learning, artificial neural networks, artificial intelligence

DOI: $10.25165 /$ j.ijabe.20201304.5501

Citation: Huang Y B. Research status and applications of nature-inspired algorithms for agri-food production. Int J Agric \& Biol Eng, 2020; 13(4): 1-9.

\section{Introduction}

Agriculture is the source of food. A supply chain is formed from agriculture to food to become increasingly important to the world with diminishing resources and ever-increasing population. To tackle the issues to ensure global food security, it is necessary to develop and apply advanced technologies such as artificial intelligence (AI) and nature-inspired computing in agricultural and food engineering and sciences. Nature-inspired computing associated algorithms have great potential to renovate the agriculture and food industry.

In the past, especially in the last decade, various new nature-inspired systems have been developed through the studies of nature-inspired algorithms with biological mimicking that have had further advances for bionics engineering ${ }^{[1-4]}$. In AI, machine learning (ML) algorithms from artificial neural networks (ANNs) have been developed to mimic the human brain with synthetic neurons ${ }^{[5]}$. The development of ANNs can be traced back to the 1940s and ANNs have been widely studied and applied to solve problems in various areas ${ }^{[6-11]}$. In the last thirty years, ANNs and other associated soft computing methods from ML have been expanded to solve problems in agriculture ${ }^{[12-14]}$. In recent years, research and applications of ML have entered the stage of deep learning (DL) with complex connections through multiple layers from and to various neurons ${ }^{[15,16]}$. DL algorithms are used to extract deep features of the target with high accuracy and robust system performance. Applications of DL in agriculture have appeared recently ${ }^{[17-22]}$. In the meantime, by being motivated from recent advanced bionics studies ${ }^{[23]}$, a number of

Received date: 2019-11-03 Accepted date: 2020-03-16

Biographies: Yanbo Huang, PhD, Lead Scientist and Agricultural Engineer, research interests: agricultural information technology. USDA-ARS Crop Production Research Unit, Stoneville, MS, USA. Tel: +1-6626865354, Email: yanbo.huang@usda.gov. nature-inspired algorithms have been developed, such as the krill herd $(\mathrm{KH})$ algorithm ${ }^{[24]}$, the artificial root foraging optimization (ARFO) algorithm ${ }^{[25]}$ and a hybrid bionic algorithm for solving the problems of parametric optimization ${ }^{[26]}$. With the development of the algorithms, implementation of them is supported by the platforms to intend for simple and quick use without spending time for implementing the algorithms from $\operatorname{scratch}^{[27,28]}$.

In this study, the development of ML algorithms from ANNs to DL is presented, summarized and discussed in conjunction with their biological connections for agricultural applications. In the meantime, the algorithms motivated by recent bionics studies are compared and discussed for their potentials for agriculture.

\section{Machine learning and artificial intelligence}

ML used to be not strictly categorized as a branch in "classic" AI. With the appearance of $\mathrm{DL}^{[15]}$, especially the success of AlphaGo with deep neural networks ${ }^{[16]}$, ML, including DL, becomes an overwhelming branch of AI. In contrast to the natural intelligence of humans and other animals, AI provides intelligence shown by machines or computerized systems with the functions of language and vision. AI, as a scientific discipline, began to develop in the 1950s as Alan Turing proposed a test called "The Imitation Game" ${ }^{,[29]}$. After twenty years Terry Winogard first operationalized Turing's intelligent machine by creating a blocks world with a natural language understanding computer program, SHRDLU $^{[30]}$. Experienced several waves of optimism and disappointment with "spring" and "winter", AI finally started a new age with ML in the 1980s while before ML, all AI systems worked with hand-designed rules, i.e. man-made rules, which was hard to anticipate all possibilities to adapt to new situations in an assumed closed world. ML develops and uses statistical techniques with the methods of pattern recognition and computational learning theory to allow computerized systems to be able to "learn", e.g. the ability to progressively improve the system performance in solving 
a specific problem with data without being explicitly modeled or programmed $^{[31,32]}$.

\section{Artificial neural networks and soft computing}

Obviously, compared with hand-designed rule AI systems, ML offers an "open-world" scheme to design and develop new-generation AI systems. The ideas of the open world with learning ability were motivated biologically by human brains. With this motivation, ML was developed into the nature-inspired paradigms or algorithms represented by ANNs.

The development of ANNs started designing the artificial neuron to mimic the characteristics of the biological neuron. The human nervous system is built of cells called neurons. Figure 1 shows the structure of a pair of typical biological neurons. In the structure, dendrites extend from the cell body of one neuron to the cell body of the other neuron and other neurons, where they neurons receive signals through synapse at the connection point. On the receiving side of the synapse, these inputs are conducted to the cell body, where the inputs are summed, some of which are tending to excite the cell while others to inhibit it to fire. When the cumulative excitation in the cell body exceeds a threshold, the cell fires and sends a signal down the axon to other neurons.

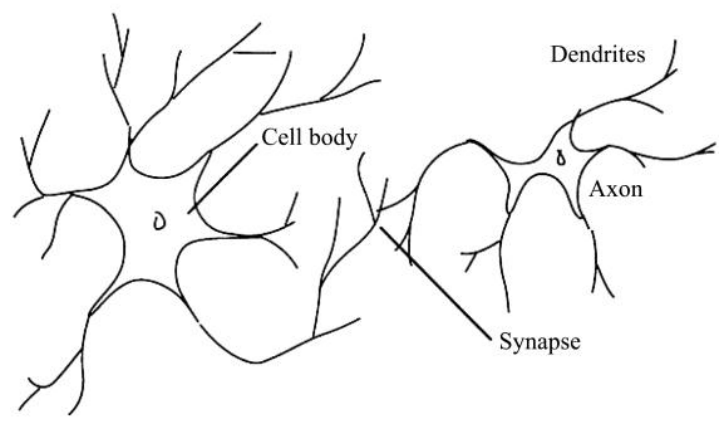

Figure 1 Biological neuron ${ }^{[33]}$

Of course, the description above is just the basic function of biological neurons. The real neurons work with many complexities and exceptions. ANNs only simplifies and models the basic functions. Figure 2 shows the structure and calculation of the artificial neuron to mimic the first-order characteristic of the biological neuron. In the artificial neuron a set of inputs, $x_{1}, x_{2}, \cdots$, $x_{n}$, are applied, each of which represents the output of another neuron. Then, each of the inputs, $x_{i}$ is multiplied by its corresponding weight, $w_{\mathrm{i}}$, which is analogous to a synaptic strength, and all the multiplications i.e. the weighted inputs, $w_{i *} * x_{i}$, are summed to determine the activation level of the neuron. With the weighted sum the output of the neuron, $z$, is produced by an activation function. The function can be a simple linear threshold function. In order to more accurately simulate the nonlinear transfer characteristics of the biological neuron a number of nonlinear activation functions were used for artificial neurons. Among them the sigmoidal function has been mostly used, which is expressed as:

$$
\Phi(y)=\frac{1}{1+e^{-y}}
$$

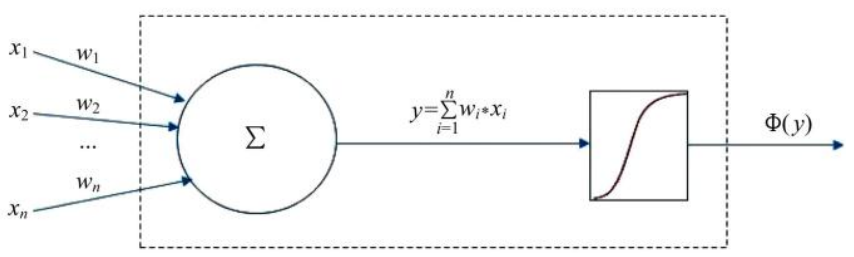

Figure 2 Artificial neuron
A group of artificial neurons are connected in a way to mimic the behavior of biological neurons in the human brain with the web of connectivity and interactivity, which formulates so-called artificial neural network (ANN). There are different ANNs with different connectivity of neurons. Figure 3 shows feedforward, recurrent and feedback, fully connected, auto-associative and hetero associative ANNs. Among them, the feedforward ANN is the most widely used in pattern recognition. In 1986 a series of results about backpropagation (BP) training algorithm for multilayer feedforward ANN was published ${ }^{[34,35]}$. In 1989 the multilayer feedforward ANN with one hidden layer was proven as the universal approximator for any continuous function ${ }^{[36,37]}$. These works lead to a wave of machine learning based on statistical models. Studies indicated that the BP algorithm could train a feedforward ANN model to learn from a quite amount of data samples to extrapolate or predict unknown events. People found that this statistics-based machine learning method has a lot of advantages over the man-made rule-based systems.
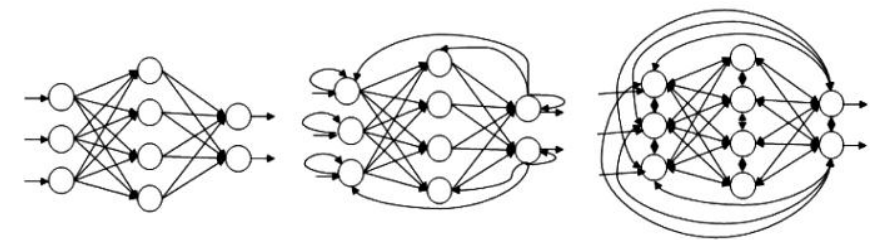

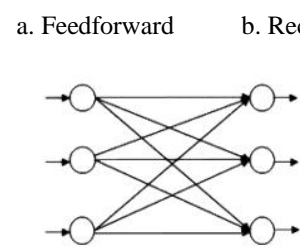

d. Auto associative c. Fully connected

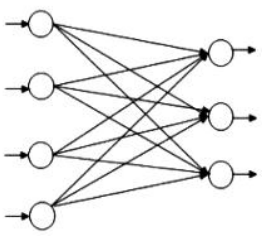

e. Hetero associative
Figure 3 Typical ANNs

The BP algorithm is a supervised learning algorithm. That is that the net optimization is based on the known desired output. However, in reality, the desired output is often unknown and the system optimization is based on self-organizing. The training algorithms in this category without known desired output are unsupervised learning algorithms, which are more and more developed and used although in 1982 Kohonen already established the self-organizing feature mapping network ${ }^{[38]}$.

With the success of the BP neural networks, radial basis function networks and support vector machine (SVM) appeared to be developed and used widely with great success as well ${ }^{[39,40]}$. Soft computing is a computational approach to learning and machine intelligence ${ }^{[41]}$. It differs from conventional (hard) computing in that it is tolerant of imprecision, uncertainty, partial truth, and approximation.

In the 1980s various rule-based expert systems were developed to bring up a wave of AI research and development. Examples are the expert systems for crop fertilization and economic forecasting method selection ${ }^{[42]}$. In the early 1990s, Kohonon self-organizing feature mapping network was originally developed and applied for unsupervised ultrasonic signal classification for beef grading ${ }^{[43]}$ and accordingly a counter propagation network to model a particleboard manufacturing process ${ }^{[44,45]}$. A Ph.D. research developed multi-layer feedforward neural networks with backpropagation training algorithm to identify the multiple input and multiple output relationship of a snack food frying process unit operation and recurrent neural network with backpropagation through time training algorithm to characterize the dynamics 
between the inputs and outputs of the unit operation, and based on modeling the neural network process models were inversed through numerical optimization to design and implement model predictive controllers to handle the nonlinearity and input-output time lags of the process ${ }^{[46-50]}$. Figure 4 shows the closed loop of neural network modeling and control for the snack food frying process unit operation. In the late 1990s for meat quality evaluation wavelet textural features were developed for quantitative ultrasonic elastographic image analysis ${ }^{[49,51]}$. With the wavelet textural features multilayer feedforward neural networks were developed through investigating the efficiency of the training processes and the generalization of the networks using the gradient descent and Levenberg-Marquardt optimization algorithms in backpropagation and weight-decay was added in the Levenberg-Marquardt backpropagation to improve the generation of the neural network models $^{[49,52]}$. In the late $2000 \mathrm{~s}$, all fundamental and associated ANN architectures and training algorithms were reviewed and the further ANN development related to support vector machine (SVM) was discussed in conjunction with applications in food science and engineering, soil and water relationship for crop management, and decision support for precision agriculture ${ }^{[12]}$. Then, ANNs were put into consideration as the major force of soft computing to emulate the human mind along with fuzzy logic, genetic algorithms, Bayesian inference, and decision tree ${ }^{[13]}$. In 2013 a group of scientists discussed challenges and issues in conducting agroecological studies from a statistical point of view, including neural networks ${ }^{[53]}$.

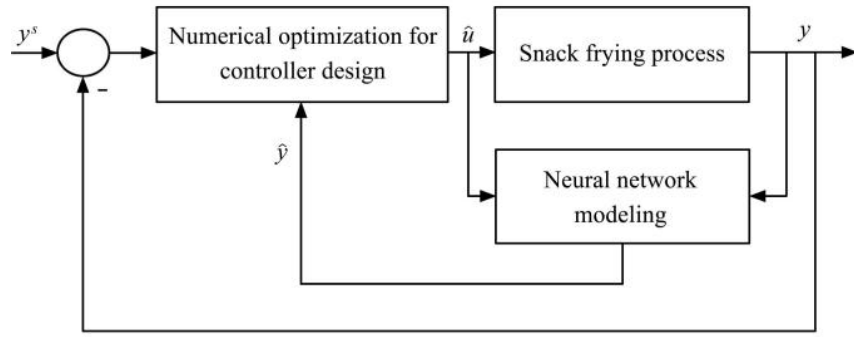

$y$ - process output vector; $\hat{y}$ - one-step ahead or multi-step ahead process output prediction vector; $y^{s}$ - process output reference vector; $\hat{u}$ - inversed process input vector from controller

Figure 4 Closed loop of neural network modeling and control for the snack food frying process unit operation

In recent years, a study was conceived and initialized a study using machine learning algorithms of naive Bayes, random forest and SVM to assess soybean injury from dicamba, an herbicide used to control broadleaf weeds in crop fields, through hyperspectral imaging ${ }^{[54]}$. Studies were conducted using machine learning algorithms of K-nearest neighbor, random forest, and a genetic algorithm coupled with an SVM to create a spectral library to enhance crop classification and growth status monitoring ${ }^{[55]}$, using K-nearest neighbor and SVM for classification of broilers to analyze their behaviors ${ }^{[56]}$, and using SVM classification of unmanned aerial vehicle (UAV) color images to monitor cotton budding $^{[57]}$.

\section{Deep learning}

ANNs before the 2000 s can be tentatively categorized as shallow learning in machine learning. Then, the most successful ANNs are the ones with the architecture of multilayer feedforward and the supervised training algorithms of BP. In 2006 Hinton and Salakhutdinov started the concepts of DL by illustrating that the ANNs with many hidden layers have strong ability of feature learning and the difficulty of deep ANNs' training can be overcome by layer-wise pre-training ${ }^{[15]}$. Although the previous ANNs mostly succeeded with supervised training, the layer-wise pre-training of Hinton and Salakhutdinov's work was conducted through unsupervised learning. However, DL did not gain its popularity until AlphaGo was announced ${ }^{[16]}$ and beat a number of top Go players in the world. From shallow ANNs to deep ANNs the network structure becomes much more complicated with a lot of more layers and neurons (Figure 5). Also, deep ANNs provide their ability to learn data representations mostly in an unsupervised manner and generalize to unseen data samples using hierarchical representations. Deep ANNs are leading another wave of machine learning to advance AI technology.
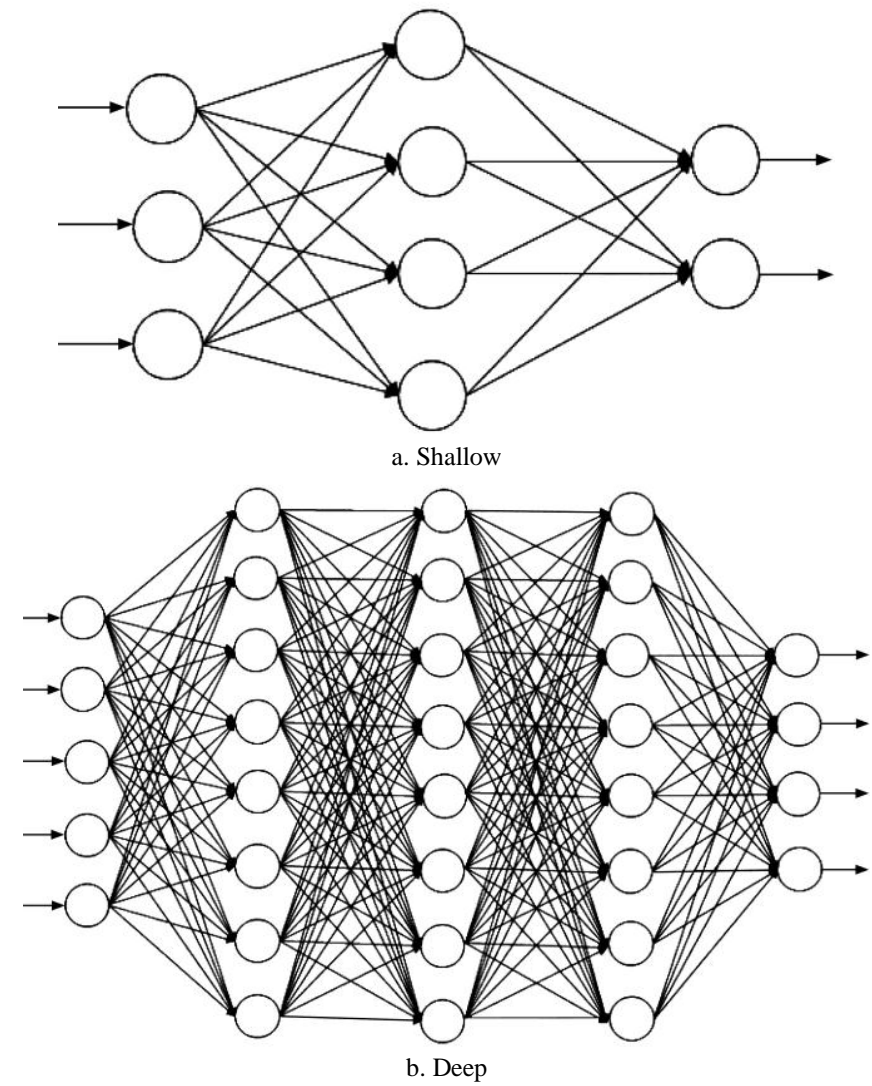

Figure 5 ANN network structure

In the past few years, DL has been rapidly studied, developed and applied ${ }^{[58]}$. There are a number of DL models such as Deep Belief Network ${ }^{[59]}$, Convolutional Neural Network $(\mathrm{CNN})^{[60]}$, and Stacked Autoencoder ${ }^{[61]}$. CNNs have transformation invariance in translation, angle of view, size, or illuminance so that they are widely used in pattern recognition and image analysis. CNNs are biologically inspired variants of multilayer perceptrons. They are designed to emulate the behavior of the visual cortex. The CNN models mitigate the challenges posed by the multilayer feedforward architecture by exploiting the strong spatially local correlation present in natural targets and images.

With these characteristics, CNNs are the most widely used deep ANNs so far. LeCun et al. (1989) first proposed a $\mathrm{CNN}^{[62]}$. This CNN was improved for hand-writing character recognition ${ }^{[63,64]}$. Until the appearance of AlexNet ${ }^{[55]}$, deep CNNs began rapid development in theoretical studies and practical applications. It is noted that this $\mathrm{CNN}$ uses a new activation function to reduce the computation, speed up training convergence and mitigate overfitting ${ }^{[65]}$ :

$$
\operatorname{ReLU}(x)=\max (0, x)
$$


where, $R e L U$ stands for rectified linear unit and its derivative is the sign function:

$$
\operatorname{ReLU}^{\prime}(x)=\left\{\begin{array}{l}
1, \text { if } x>0 \\
0, \text { otherwise }
\end{array}\right.
$$

AlexNet was modified and improved with $\mathrm{ZFNet}^{[66]}$ and GoogLeNet ${ }^{[67]}, \mathrm{VGG}^{[68]}$, residual network ${ }^{[69]}$ and their variants advanced greatly deep CNN techniques. Currently, the studies are being conducted to improve deep CNNs and optimize their training processes in the convolution layer, pooling layer, activation function, loss function, network architecture and data regularization with the structure of a typical $\mathrm{CNN}$ as shown in Figure 6.

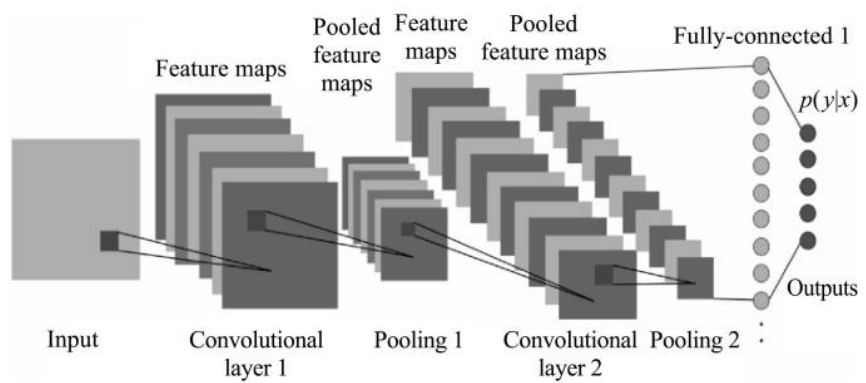

Figure 6 Structure of a CNN, consisting of convolutional, pooling, and fully-connected layers ${ }^{[70]}$

In recent years more and more journal articles on DL have been published. Figure 7 shows the journal publications in the world on DL up to 2015. In 2017 AlphaGo Zero was announced $^{[71]}$ by advancing AlphaGo by learning from scratch to totally beat AlphaGo which strongly depends on prior human knowledge, which preludes a new wave of deep learning research, development and applications.
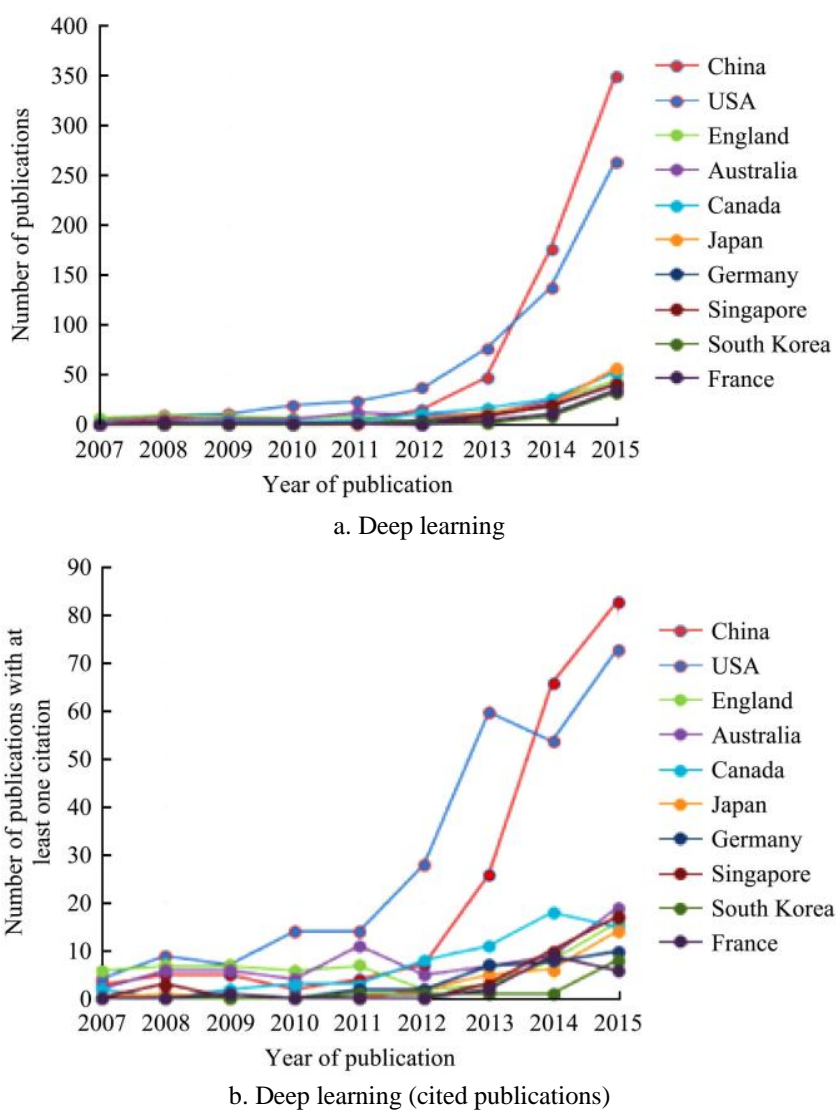

Source: US Office of Science and Technology Policy/White House

Figure 7 (a) Journal articles mentioning "deep learning" or "deep neural network" by nation; (b) Journal articles cited at least once, mentioning "deep learning" or "deep neural network", by countries
DL requires massive computation. The web network structures of deep ANNs need a graphic processing unit (GPU) specialized for high-performance computing. With the rapid development of DL and AI, a number of GPU-based DL computing frameworks have been created for high-performance DL system computing and operation. The examples include TensorFlow (Google), Theano (Université de Montréal), PyTorch (Facebook), Torch (New York University/Facebook) and Caffe (University of California, Berkeley). The tensor processing unit (TPU) is a chip designed by Google for machine learning workloads. Compared to GPU TPU has faster speed and much more memory. Actually, TPU, GPU and even CPU all can be used for deep learning. However, which DL models fit each of TPU, GPU and CPU, respectively, is an issue to consider. Wang et al. (2019) ${ }^{[72]}$ designed a type of software named ParaDnn for benchmark testing of DL parametrization to help determine TPU, GPU, or CPU for different DL models.

The success of DL is built on a great amount of data and the state-of-the-art supercomputing power allows training of scalable large neural networks for better performance with more data than shallow ANNs in ML that will stay with more data. However, the amount of data in reality often could not match the algorithm to be successful. In the case of limited data more data have to be created by augmenting the limited data, for example, turn, shift, scale, rotate and cut an image. Data augmentation can be done offline for relatively small data sets and online dynamically in the computing program for relatively large data sets.

With the development of ML new models have been developed to improve and enhance general ML and DL training and analysis. Examples are the general adversarial network $(\mathrm{GAN})^{[73]}$, deep transfer learning ${ }^{[74]}$, and AutoML ${ }^{[75]}$.

DL has been widely developed and applied in agriculture to expect the improved performance of monitoring, estimation and analysis. Various DL architectures and models have been used in agriculture and $\mathrm{CNN}$ is dominated in most of the research and applications $^{[19,20,76]}$.

Deep CNN was tested for classifying the images of corn, cotton and soybean leaves collected in the fields. Nine leaves of each crop were collected from the fields and half of them were kept in coolers and half were left in the lab room, respectively, within $1 \mathrm{~d}$ after the field collection and the images were taken with a portable digital camera with the time within $24 \mathrm{~h}$ at $15 \mathrm{~m}, 1 \mathrm{~h}, 2 \mathrm{~h}$, $3 \mathrm{~h}, 4 \mathrm{~h}, 5 \mathrm{~h}, 6 \mathrm{~h}$, and $24 \mathrm{~h}$, which generated 144 images of each crop. Figure 8 shows two representative leaf images of each crop over the time within $24 \mathrm{~h}$. The purpose of the test was to classify the leaves regardless of the time during $24 \mathrm{~h}$. In the project, a CNN was created (Figure 9 and Table 1) using TensorFlow in a Python NumPy program (NumPy_CNN.py calls TensorFlow_leaves.py) to conduct leaf classification. With data augmentation, each crop was doubled with the images, half for training and a half for testing of the CNN network. Figure 10 illustrates the implementation of the $\mathrm{CNN}$ for leaf classification. Table 2 is the confusion matrix of $\mathrm{CNN}$ testing results for leaf classification, which indicates that the $\mathrm{CNN}$ can classify the leaves of the three crops regardless of the time during $24 \mathrm{~h}$ when the vigor of the leaves decayed in general but the model still can be adjusted to further improve the classification, which may indicate that straight use of CNN might not be suitable for this problem, and a more suitable DL scheme may be needed. At present CNN almost becomes the synonym of DL for new people in this field. A lot of applications claim the use of DL to simply apply CNN. However, 
the straight use of CNN often cannot achieve what expected from DL for a lot of problems. When solving a problem, the specific characteristics of the problem should be carefully identified and analyzed. On this basis, a "use-inspired" DL approach can be developed to seek a most suitable method and understanding in DL by situating the research in a domain of application to simultaneously inform progress in DL and solve problems in particular use cases. This is what this research doing to advance the DL research to solve this specific problem and other similar problems at the same time to not only classify the leaves regardless of the time but also further detect the change of the leaves with time with deep feature extraction to formulate the time series of the leaf images.

Recently CNNs were evaluated for cage-free floor egg detection $^{[77]}$. This study developed vision-based floor-egg detectors using three variants of $\mathrm{CNN}$, single shot detector, faster region-based $\mathrm{CNN}$ (faster $\mathrm{R}-\mathrm{CNN}$ ), and region-based fully convolutional network (R-FCN), which have been widely used for object detection and recognition ${ }^{[78,79]}$, and the three detectors were evaluated their performance on floor egg detection under simulated

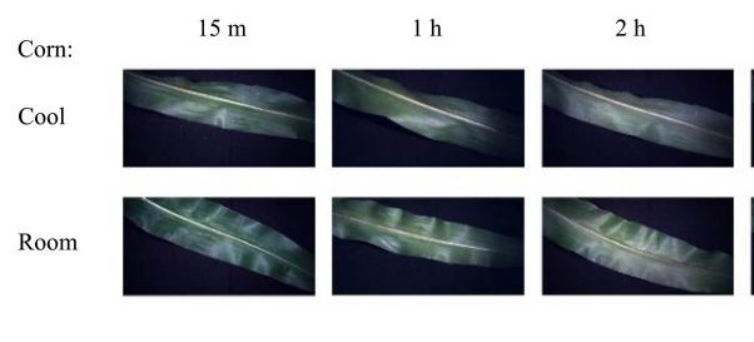

$3 \mathrm{~h}$
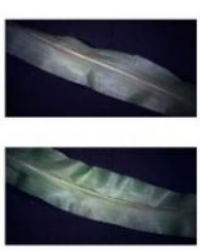

cage-free environments.

Table 1 CNN architecture for leaf classification

\begin{tabular}{ccc}
\hline Layer & Kernel Size & Output \\
\hline Input & - & $420 \times 420 \times 3$ \\
Resize & $128 \times 128 \times 3$ & $128 \times 128 \times 3$ \\
Conv 1 & $3 \times 3 \times 32$ & $128 \times 128 \times 32$ \\
Pool 1 & $2 \times 2 \times 1$ & $64 \times 64 \times 32$ \\
Relu 1 & - & $64 \times 64 \times 32$ \\
Conv 2 & $3 \times 3 \times 32$ & $64 \times 64 \times 32$ \\
Pool 2 & $2 \times 2 \times 1$ & $32 \times 32 \times 32$ \\
Relu 2 & - & $32 \times 32 \times 32$ \\
Conv 3 & $3 \times 3 \times 64$ & $32 \times 32 \times 64$ \\
Pool 3 & $2 \times 2 \times 1$ & $16 \times 16 \times 64$ \\
Relu 3 & - & $16 \times 16 \times 64$ \\
Flat 1 & - & $1 \times 1 \times 16384$ \\
Fc 1 & $1 \times 1 \times 256$ & $1 \times 1 \times 256$ \\
Relu 4 & - & $1 \times 1 \times 256$ \\
Fc 2 & $1 \times 1 \times 6$ & $1 \times 1 \times 6$ \\
Softmax & - & $1 \times 1 \times 6$ \\
\hline
\end{tabular}

Cotton:

Cool
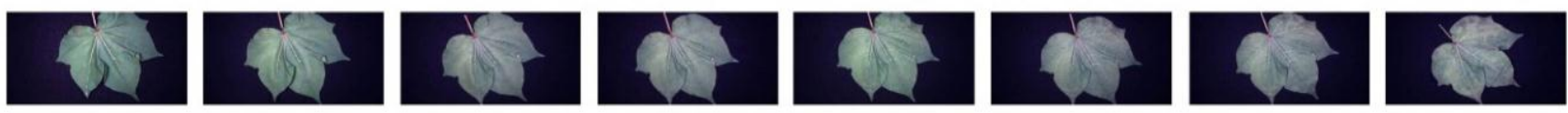

Room
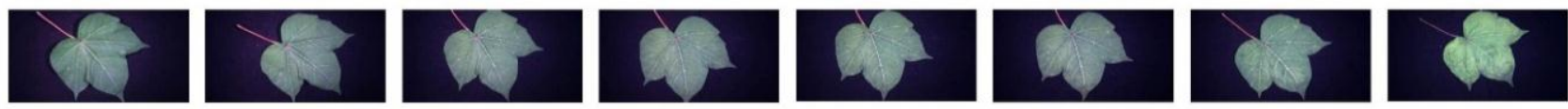

Soybean:
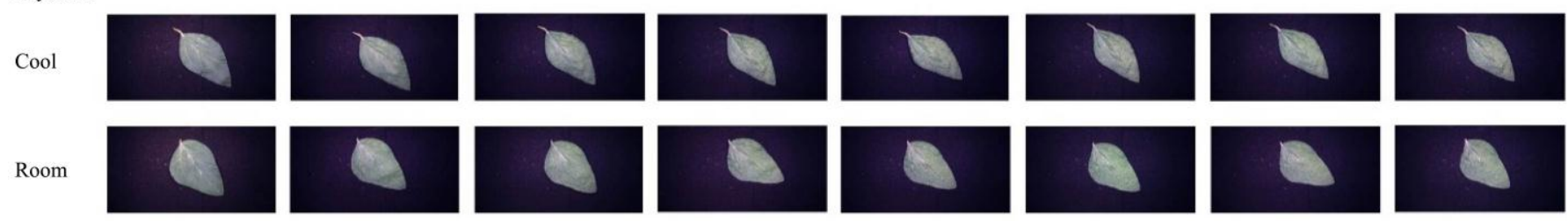

Figure 8 Two representative leaf images of each crop over the time within the $24 \mathrm{~h}$

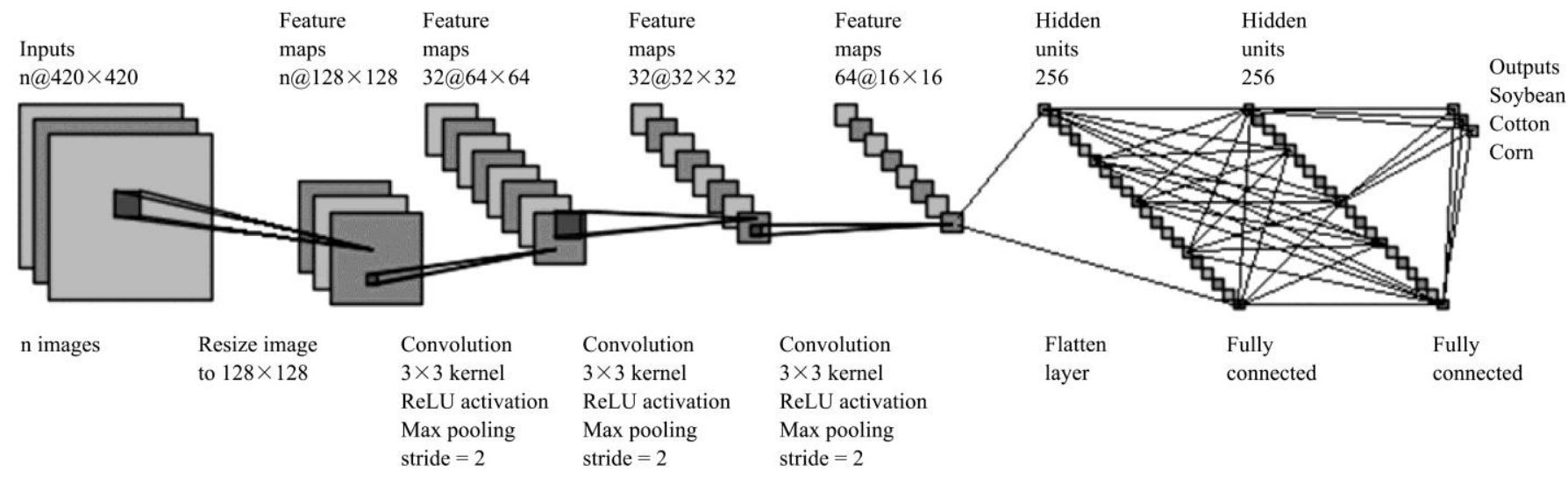

Figure $9 \mathrm{CNN}$ for leaf classification 


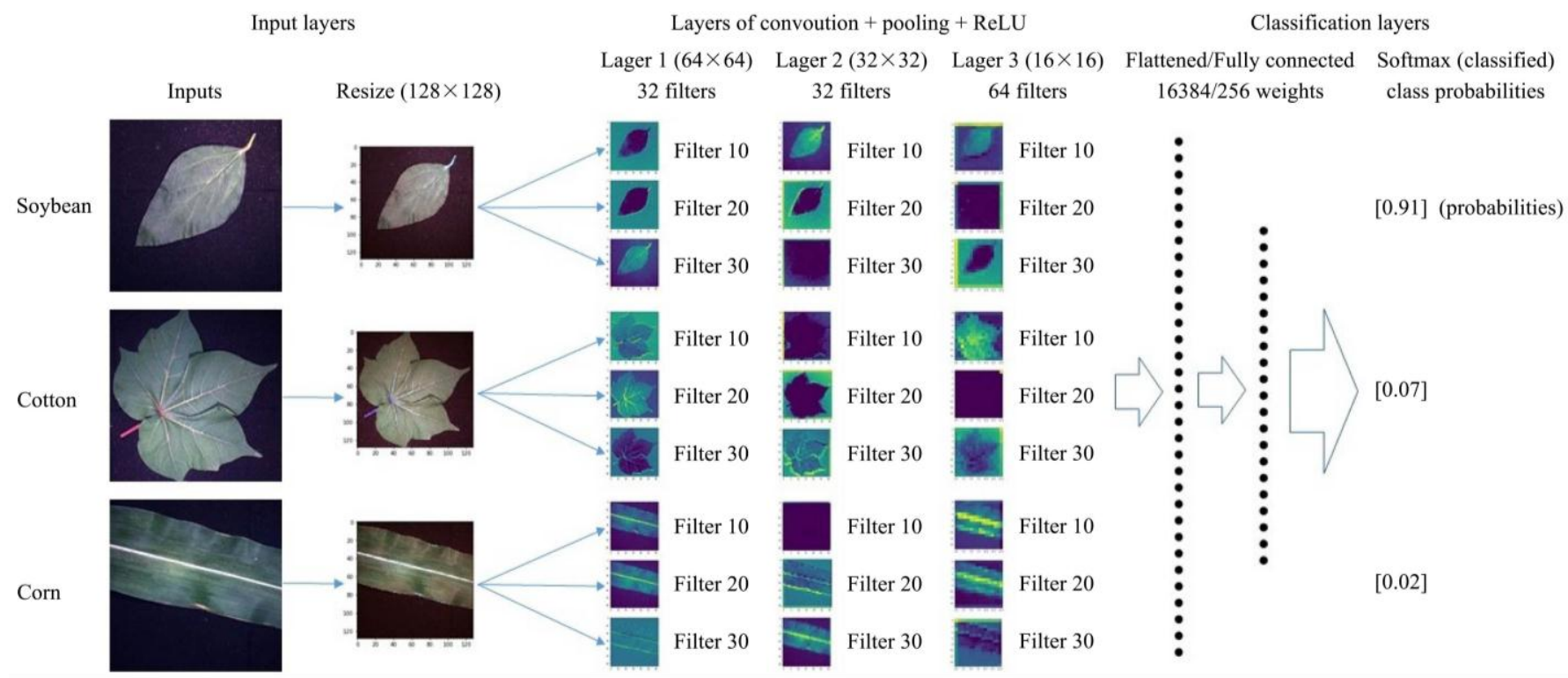

Figure 10 Implementation of the $\mathrm{CNN}$ for leaf classification

Table 2 Confusion matrix of CNN testing for leaf classification

\begin{tabular}{cccc}
\hline \multirow{2}{*}{ Actual } & \multicolumn{3}{c}{ Classified } \\
\cline { 2 - 4 } & Corn & Cotton & Soybean \\
\hline Corn & $100(69 \%)$ & $12(8 \%)$ & $32(22 \%)$ \\
Cotton & $40(28 \%)$ & $92(64 \%)$ & $12(8 \%)$ \\
Soybean & $4(3 \%)$ & $40(28 \%)$ & $100(69 \%)$ \\
\hline
\end{tabular}

\section{Bionics motivated algorithms}

Bionics is a scientific discipline that investigates to apply nature-inspired biological methods and systems to the study and design of advanced technology and engineering systems. The word, Bionics, was coined by Dr. Jack E. Steele in 1958 with meaning "like life" when he was working at the Aeronautics Division House at Wright-Patterson Air Force Base in Dayton, Ohio. Bionics engineering is to develop and implement the advanced technology and engineering systems from bionics studies. Classic examples of bionics in engineering include sonar, radar, and ultrasound imaging imitating animal echolocation.

With the development of bionics more and more algorithms have been developed for complex computational applications by getting ideas from observing how nature behaves to solve complex problems. Although compared to ML the research on designing and developing nature-inspired algorithms is still very young, there are some successful nature inspired computing and complex systems for understanding and designing more such systems with novelty in AI. Nature-inspired algorithms are principal among metaheuristic algorithms that are found to be more powerful than the conventional methods that are based on formal logic or mathematical programming ${ }^{[00,81]}$.

Ganomi and Alavi (2012) ${ }^{[82]}$ illustrated that a group of algorithms inspired by biology have been developed and are divided into three main categories ${ }^{[83]}$ :

(1) Evolutionary algorithms,

(2) Swarm intelligence, and

(3) Bacterial foraging algorithms.

The evolutionary algorithms are inspired by the genetic evolution process. Among them, a genetic algorithm $(\mathrm{GA})^{[84]}$, as mentioned above as a soft computing technique, is the most used one. Others include genetic programming $(\mathrm{GP})^{[85]}$, evolutionary strategy $(\mathrm{ES})^{[86]}$ and differential evolution $(\mathrm{DE})^{[87]}$. These population-based stochastic search algorithms work with best-to-survive criteria to optimize. The evolutionary algorithms have been remarkably improved over the last decades. Examples are Stud genetic algorithm $(\mathrm{SGA})^{[88]}$ and multi-stage genetic programming ${ }^{[82]}$ for improved GP non-linear system modeling. In 2008, Simon ${ }^{[89]}$ has proposed a new evolutionary algorithm, namely biogeography-based optimization (BBO). The BBO algorithm is used for global recombination and uniform crossover which are inspired by the GA literature.

In swarm intelligence particle swarm optimization (PSO) ${ }^{[90]}$ and ant colony optimization $(\mathrm{ACO})^{[91]}$ are well known. These algorithms are based on the simulation of the collective behavior of animals. PSO is a population-based method inspired by the social behavior of bird flocking or fish schooling. The ACO algorithm is inspired by the collective foraging behavior of ants.

The bacterial foraging algorithms emulate the bacterial foraging behavior for new bio-inspired optimization approaches ${ }^{[83,92]}$. Examples are computing systems of microbial interactions and communications (COSMIC) ${ }^{[93]}$ and rule-based bacterial modeling (RUBAM) ${ }^{[94]}$.

However, these nature-inspired metaheuristic methods have the problem of the parameter adaptation. Valdez ${ }^{[3]}$ surveyed mainly the PSO, Gravitational Search (GS), and ACO algorithms for modifications using fuzzy logic to solve this problem and obtain better results than the original methods. A study was conducted to use a multi-layer perceptron and PSO in modeling and predicting the germination rate of two common bean cultivars as a function of distinct temperatures ${ }^{[95]}$.

Other bio-inspired algorithms include, but not limited to, bees algorithm $^{[96]}$, firefly algorithm ${ }^{[1,80,97]}$, krill herd algorithm ${ }^{[24]}$, artificial root foraging optimization (ARFO) algorithm ${ }^{[25]}$.

\section{Agricultural perspectives of the discussed algorithms}

ANNs and associated soft computing techniques have been widely used in crop production management, irrigation management, soil analysis, precision agricultural system integration and pesticide application control ${ }^{[13]}$. Deep learning has been 
developed for agriculture uses, especially for identification of weeds, land cover classification, plant recognition, fruits counting and crop type classification ${ }^{[20]}$. Metaheuristic algorithms have been developed for agricultural land use optimization in economic crop planning, water resources management, nature conservation in the landscape, and multifunctional agricultural landscape using genetic algorithms, $\mathrm{PSO}, \mathrm{ACO}$, etc. ${ }^{[98]}$. It can be believed that with the requirement to advanced agricultural operations into intelligent and automatic smart stages nature-inspired algorithms will play more and more important roles for sustainable agri-food production.

However, quite often complicated methods do not necessarily work better. Each application has different characteristics and each algorithm has its own limitation no exception of naturally-inspired algorithms. For example, DL is limited in high data and computing requirements and poor interpretability. In practice, the selection of data analysis methods should be based on the characteristics of data and application. Confirmation of the validity of the results is essential to all methods. Any research that proposes to use naturally-inspired algorithms requires sufficient justification for compelling reasons to use these algorithms over other methods and conducts a benchmark comparison to show naturally-inspired algorithms are better; otherwise, this research is only one-sided and biased and hard to make the sense in practical application.

\section{Conclusions}

In this study, the research status and applications of nature-inspired algorithms, including ML algorithms from ANNs to DL and the algorithms motivated by bionics studies, are presented, summarized and discussed in conjunction with their biological connections for agri-food systems applications. Within the context, with recent progress reports, classic literature are also included and analyzed to provide insight into the roots of the technologies to capture the intricate nature of the technologies.

With the coming spring of AI, it can be believed that ML, especially DL algorithms, will be greatly studied, developed and applied for solving problems in various areas of interests, including in agri-food system analysis for decision support. Agri-food system environment and management are dominated by uncertainty with complex interactions of various uncontrollable factors. This paper is expected to offer a headstart for scientists and engineers not only to use current science-based nature-inspired algorithms but also to develop use-inspired algorithms with the problems in agri-food areas to deal with those issues conventional approaches cannot solve well.

\section{[References]}

[1] Zang H N, Zhang S J, Hapeshi K. A review of nature-inspired algorithms. Journal of Bionic Engineering, 2010; 7(Supplement): S232-S237.

[2] Fister I J, Yang X S, Fister I, Brest J, Fister D. A brief review of nature-inspired algorithms for optimization. Elektrotehniski Vestnik (English Edition), 2013; 80(3): 1-7.

[3] Valdez F, Melin P, Castillo O. A survey on nature-inspired optimization algorithms with fuzzy logic for dynamic parameter adaptation. Expert Systems with Applications, 2014; 41(14): 6459-6466.

[4] Speck O, Speck D, Horn R, Gantner J, Sedlbauer K P. Biomimetic bio-inspired biomorph sustainable? An attempt to classify and clarify biology-derived technical developments. Bioinspiration \& Biomimetics, 2017; 12(1): 011004. doi: 10.1088/1748-3190/12/1/011004

[5] Wasserman P D. Neural computing: Theory and practice. New York: Van Nostrand Reinhold, 1989; $230 \mathrm{p}$.

[6] McCulloch W S, Pitts W. A logical calculus of the ideas immanent in nervous activity. Bulletin of Mathematical Biophysics, 1943; 5: 115-133.
[7] Hebb D O. The organization of behavior: A neuropsychological theory. New York: John Wiley and Sons, 1949; 335p.

[8] Rosenblatt F. The perceptron: A probabilistic model for information storage and organization in the brain. Psychological Review, 1958; 65: 386-408.

[9] Minsky M, Papert S. Perceptrons: An introduction to computational geometry. Cambridge, MA: MIT Press, 1969; 308p.

[10] Grossberg S. Adaptive pattern classification and universal recoding. Biological Cybernetics, 1976; 23: 121-134.

[11] Hopfield J J. Neural networks and physical systems with emergent collective computational abilities. Proc. Natl Acad. Sci., 1982; 79: 2554-2558.

[12] Huang Y B. Advances in artificial neural networks - Methodological development and application. Algorithms, 2009; 2(3): 973-1007.

[13] Huang Y B, Lan Y B, Thomson S J, Fang A, Hoffmann W C, Lacey R Development of soft computing and applications in agricultural and biological engineering. Computers \& Electronics in Agriculture, 2010; 71(2): 107-127.

[14] Liakos K G, Busato P, Moshou D, Pearson S, Bochtis D. Machine learning in agriculture: A review. Sensors, 2018; 18(8): 2674. doi: $10.3390 / \mathrm{s} 18082674$

[15] Hinton G E, Salakhutdinov R R. Reducing the dimensionality of data with neural networks. Science, 2006; 313(5786): 504-507.

[16] Silver D, Huang A, Maddison C J, Guez A, Sifre L, van den Driessche G, et al. Mastering the game of Go with deep neural networks and tree search. Nature, 2016; 529: 484-489.

[17] Mohanty S P, Hughes D, Salathe M. Using deep learning for image-based plant disease detection. Front. Plant Sci., 2016; 7: 1419. doi: 10.3389/fpls.2016.01419.

[18] Sladojevic S, Arsenovic M, Anderla A, Culibrk D, Stefanovic D. Deep neural networks based recognition of plant diseases by leaf image classification. Comput. Intell. Neurosci., 2016; 3289801. doi: $10.1155 / 2016 / 3289801$

[19] Kussul N, Lavreniuk M, Skakun S, Shelestov A. Deep learning classification of land cover and crop types using remote sensing data. IEEE Geoscience and Remote Sensing Letters, 2017; 14(5): 778-782.

[20] Kamilaris A, Prenafeta-Boldú F X. Deep learning in agriculture: A survey. Computers and Electronics in Agriculture, 2018; 147(1): 70-90.

[21] Barbedo J G A. Plant disease identification from individual lesions and spots using deep learning. Biosystems Engineering, 2019; 180: 96-107.

[22] Barbedo J G A. Impact of dataset size and variety on the effectiveness of deep learning and transfer learning for plant disease classification. Computers and Electronics in Agriculture, 2018; 153: 46-53.

[23] Zang H, Zhang S, Hapeshi K. A review of nature-inspired algorithms. Journal of Bionic Engineering, 2010; 7: S232-S237.

[24] Gandomi A H, Alavi A H. Krill herd: A new bio-inspired optimization algorithm. Commun. Nonlinear Sci. Numer. Simulat., 2012; 17(12): 4831-4845.

[25] Ma L, Zhu Y, Liu Y, Tian L, Chen H. A novel bionic algorithm inspired by plant root foraging behaviors. Applied Soft Computing, 2015; 37: 95-113.

[26] Zaporozhets D Y, Zaruba D V, Kureichik V V. Hybrid bionic algorithms for solving problems of parametric optimization. World Applied Sciences Journal, 2013; 23(8): 1032-1036.

[27] Kapur R. Review of nature inspired algorithms in cloud computing. International Conference on Computing, Communication \& Automation, Noida: IEEE, 2015; pp. 589-594.

[28] Vrbančič G, Brezočnik L, Mlakar U, Fister D, Fister J I. NiaPy: Python microframework for building nature-inspired algorithms. The Journal of Open Source, 2018; 3(23): 613. doi: 10.21105/joss.00613.

[29] Turing A M. Computing machinery and intelligence. Computers and Thought, 1950; 49: 433-460.

[30] Winogard, T. Procedures as a representation for data in a computer program for understanding natural language. $\mathrm{PhD}$ dissertation. Cambridge, MA: Massachusetts Institute of Technology, 1971, 2. 464 p.

[31] Samuel A L. Some studies in machine learning using the game of checkers. IBM Journal of Research and Development, 1959; 3(3): 211-299.

[32] Samuel A L. Some studies in machine learning using the game of checkers. I. In: Levy D N L (Ed.). Computer Games I. New York: Springer, 1988; pp.335-365.

[33] Doszkocs T E. Neural networks in libraries: The potential of a new information technology. Avian Diseases, 1991. doi: 
10.1637/7163-020204R

[34] Rumelhart D E, McClelland J L. Parallel distributed processing: Explorations in the microstructures of cognition. Vols. I \& II. Cambridge: MIT Press, 1986; 1199p.

[35] Rumelhart D E, Hinton G E, Williams R J. Learning representation by back-propagating errors. Nature, 1986; 323(9): 533-536.

[36] Hornik K, Stinchcombe M, White H. Multilayer feedforward neural networks are universal approximators. Neural Networks, 1989; 2: 359-366.

[37] Cybenko G. Approximator by superpositions of a sigmoidal function. Math. Control Signals Systems, 1989; 2: 303-314.

[38] Kohonen T. Self-organized formation of topologically correct feature maps. Biological Cybernetics, 1982; 43(1): 59-69.

[39] Chen S, Cowan C F N, Grant P M. Orthogonal least squares learning algorithm for radial basis function networks. IEEE Transactions on Neural Networks, 1991; 2(2): 302-309.

[40] Suykens J A K, Vandewalle J. Least squares support vector machine classifiers. Neural Processing Letters, 1999; 9: 293-300.

[41] Jang J S R, Sun C T, Mizutani E. Neuro-fuzzy and soft computing: A computational approach to learning and machine intelligence. Englewood Cliffs: Prentice-Hall, 1997; 652p.

[42] Wang Y L, Huang Y B. Designing and developing expert system for selecting economic forecasting method. Journal of North China University of Technology, 1989; (1): 87-96. (in Chinese)

[43] Whittaker A D, Park B, McCauley J D, Huang Y B. Ultrasonic signal classification for beef quality grading through neural networks. Proceedings of the Automated Agriculture for the 21st Century Symposium, Chicago: ASAE, 1991, 12. pp.16-17.

[44] Whittaker A D, Huang Y B, Cook D F. Counter propagation for modeling of a continuous correlated process. American Society of Agricultural Engineers, St. Joseph: ASAE, 1992; 927060. 10.

[45] Cook D F, Whittaker A D, Huang Y. Neural network process modeling of particleboard manufacturing. American Society of Agricultural Engineers, St. Joseph: ASAE, 1992; 927061. 9.

[46] Huang Y B. Snack food frying process input-output modeling and control through artificial neural networks. Ph.D. dissertation, Texas A\&M University, College Station, TX, 1995; 184p.

[47] Huang Y B, Whittaker A D, Lacey R E. Neural network prediction modeling for a continuous snack food frying process. Transactions of the ASAE, 1998; 41(5): 1511-1517.

[48] Huang Y B, Whittaker A D, Lacey R E. Internal model control for a continuous snack food frying process using neural networks. Transactions of the ASAE, 1998; 41(5): 1519-1525.

[49] Huang Y, Whittaker A D, Lacey R E. Automation for food engineering: Food quality quantization and process control. CRC series in Contemporary Food Science. Boca Raton: CRC Press LLC, 2001; 212p.

[50] Huang Y B. Automatic process control for the food industry: an introduction. In: Caldwell D G (Ed.). Robotics and automation in the food industry. Cambridge: Woodhead Publishing, 2003; pp.4-20.

[51] Huang Y B, Lacey R E, Whittaker A D, Miller R K, Moore L, Ophir J. Wavelet textural features from ultrasonic elastograms for meat quality prediction. Transactions of the ASAE, 1997; 40(6): 1741-1748.

[52] Huang Y B, Lacey R E, Whittaker A D. Neural network prediction modeling based on ultrasonic elastograms for meat quality evaluation. Transactions of the ASAE, 1998; 41(4): 1173-1179.

[53] Bianconi A, Dalgaard T, Manly B F, Govone J J, Watts M J, Habermann G, et al. Methodological difficulties of conducting agroecological studies from a statistical perspective. Journal of Sustainable Agriculture, 2013; 37(4): 485-506

[54] Zhang J C, Huang Y B, Reddy K N, Wang B. Assessing crop damage from dicamba on non-dicamba-tolerant soybean by hyperspectral imaging through machine learning. Pest Management Science, 2019; 75: 3260-3272.

[55] Zhang J C, He Y, Yuan L, Liu L, Zhou X, Huang Y B. Machine learning-based spectral library for crop classification and status monitoring. Agronomy, 2019; 9(496): 1-17.

[56] Yang X, Zhao Y, Street G M, Huang Y. Classification of broiler behaviors using triaxial accelerometer and machine learning. International Symposium on Animal Environment and Welfare, Chongqing, 2019.

[57] Xia L, Zhang R, Chen L, Huang Y, Xu G, Wen Y, et al. Monitor cotton budding using SVM and UAV images. Applied Sciences, 2019; 9(20): 4312. doi: 10.3390/app9204312.
[58] Zhu N, Liu X, Liu Z, Hu K, Wang Y, Tan J, et al. Deep learning for smart agriculture: Concepts, tools, applications, and opportunities. Int $\mathrm{J}$ Agric \& Biol Eng, 2018; 11(4): 32-44.

[59] Hinton G E, Osindero S, Teh Y. A fast learning algorithm for deep belief nets. Neural Comput., 20166; 18(7): 1527-1554.

[60] Krizhevsky A, Sutskever I, Hinton G E. ImageNet classification with deep convolutional neural networks. Proc. Neural Inf. Process. Syst., 2012; 25(2):1-9. doi: 10.1145/3065386.

[61] Bengio Y, Lamblin P, Popovici D, Larochelle H. Greedy layer-wise training of deep networks. Proc. Neural Inf. Process. Syst., Cambridge, MA, USA. 2007; pp.153-160.

[62] LeCun Y, Boser B, Denker J S, Henderson D, Howard R E, Hubbard W, et al. Backpropagation applied to handwritten zip code recognition. Neural Computation, 1989; 1(4): 541-551.

[63] LeCun Y, Boser B, Denker J S, Henderson D, Howard R E, Hubbard W, et al. Handwritten digit recognition with a back-propagation network. In: David Touretzky, editor. Advances in neural information processing systems, 2 (NIPS*89), Denver: Morgan Kaufman. 1990; pp.396-404.

[64] LeCun Y, Bottou L, Bengio Y, Haffner P. Gradient-based learning applied to document recognition. Proceedings of the IEEE, 1998; 86(11): 2278-2324.

[65] Nair V, Hinton G E. Rectified linear units improve restricted Boltzmann machines. In: L. Bottou and M. Littman, editors, Proceedings of the Twenty-Seventh International Conference on Machine Learning, Haifa, Israel. 2010

[66] Zeiler M D, Fergus R. Visualizing and understanding convolutional networks. In: Fleet D, Pajdla T, Schiele B, Tuytelaars T (Eds). Computer Vision - ECCV 2014. Lecture Notes in Computer Science, Berlin: Springer, 2014; 8689: 818-833.

[67] Szegedy C, Liu W, Jia Y, Sermanet P, Reed S, Anguelov D, et al. Going deeper with convolutions. IEEE Conference on Computer Vision and Pattern Recognition, Boston: IEEE, 2015; pp.1-9.

[68] Simonyan K, Zisserman A. Very deep convolutional networks for large-scale image recognition. arXiv technical report, 2014. arXiv: 1409.1556.

[69] He K M, Zhang X Y, Ren S Q, Sun J. Deep residual learning for image recognition. IEEE Conference on Computer Vision \& Pattern Recognition, Las Vegas: IEEE, 2016; pp.770-778.

[70] Albelwi S, Mahmood A. A framework for designing the architectures of deep convolutional neural networks. Entropy, 2017; 19(242): 1-20.

[71] Silver D, Schrittwieser J, Simonyan K, Antonoglou I, Huang A, Guez A, et al. Mastering the game of Go without human knowledge. Nature, 2017; 550: 354-359.

[72] Wang Y E, Wei G Y, Brooks D. Benchmarking TPU, GPU, and CPU platforms for deep learning. arXiv, 2019. arXiv:1907.10701.

[73] Goodfellow I J, Pouget-Abadie J, Mirza M, Xu B, Warde-Farley D, Ozair $\mathrm{S}$, et al. Generative adversarial nets. arXiv e-print, 2014. arXiv: 1406.2661v1.

[74] Tan C, Sun F, Kong T, Zhang W, Yang C, Liu C. A survey on deep transfer learning. In: Kurkova V, Manolopoulos Y, Hammer B, Iliadis L, Maglogiannis I (Ed.). Artificial neural networks and machine learning-ICANN 2018. Cham: Springer, 2018; 11141. doi: 10.1007/978-3-030-01424-7_27.

[75] He X, Zhao K, Chu X. AutoML: A survey of the state-of-the-art. arXiv preprint, 2020. arXiv:1908.00709.

[76] Grinblat G L, Uzal L C, Larese M G, Granitto P M. Deep learning for plant identification using vein morphological patterns. Computers and Electronics in Agriculture, 2016; 127: 418-424.

[77] Li G, Xu Y, Zhao Y, Du Q, Huang Y. Evaluating convolutional neural networks for cage-free floor egg detection. Sensors, 2020; 20(2): 332. doi: 10.3390/s20020332.

[78] Wu X W, Sahoo D, Hoi C H. Recent advances in deep learning for object detection. 2019. https://arxiv.org/pdf/1908.03673.pdf. Accessed on [2019-08-10].

[79] Zhao Z, Zheng P, Xu S, Wu X. Object detection with deep learning: a review. IEEE Transactions on Neural Networks and Learning Systems, 2019; 30(11): 3212-3232.

[80] Yang X. Nature-inspired metaheuristic algorithms. Beckington: Luniver Press, 2008; 128p.

[81] Talbi E G. Metaheuristics: From design to implementation. Hoboken: John Wiley \& Sons, 2009; 624p.

[82] Gandomi A H, Alavi A H. Multi-stage genetic programming: A new strategy to nonlinear system modeling. Inf. Sci., 2011; 181(23): 5227-5239. 
[83] Tang W J, Wu Q H. Biologically inspired optimization: A review. Trans Inst Meas Control, 2009; 31: 495-515.

[84] Goldberg D E. Genetic algorithms in search, optimization and machine learning. Reading: Addison-Wesley, 1989; 432p.

[85] Koza J R. Genetic programming: On the programming of computers by natural selection. Cambridge: MIT Press, 1992.840p.

[86] Fogel L J, Owens A J, Walsh M J. Artificial intelligence through simulated evolution. Chichester: John Wiley \& Sons, Ltd, 1966; 162p.

[87] Storn R, Price K V. Differential evolution: A simple and efficient heuristic for global optimization over continuous spaces. J Global Optim. 1997; 11(4): 341-359.

[88] Khatib W, Fleming P. The stud GA: A mini revolution? In: Eiben A, Back T, Schoenauer M, Schwefel H, editors. Parallel problem solving from nature. New York: Springer. 1998. doi: 10.1007/BFb0056910.

[89] Simon D. Biogeography-based optimization. IEEE Trans Evolut Comput., 2008; 12: 702-713.

[90] Eberhart R C, Kennedy J. A new optimizer using particle swarm theory. In: Proceedings of the sixth international symposium on micro machine and human science. Nagoya: IEEE, 1995; pp.39-43.

[91] Dorigo M, Maniezzo V, Colorni A. The ant system: optimization by a colony of cooperating agents. IEEE Trans Syst Man Cybern B, 1996; 26(1): 29-41.

[92] Passino K M. Biomimicry of bacterial foraging for distributed optimization and control. IEEE Control Syst. Mag., 2002; 22: 53-67.

[93] Gregory R, Paton R C, Saunders J R, Wu Q H. Parallelising a model of bacterial interaction and evolution. BioSystems, 2004; 76: 121-31.

[94] Vlachos C, Paton R C, Saunders J R, Wu Q. A rule-based approach to the modelling of bacterial ecosystems. BioSystems, 2005; 84: 49-72.

[95] Bianconi A, Watts M J, Huang Y, Serapião A B S, Govone J S, Mi X, et al. Applying computational intelligence methods to modeling and predicting common bean germination rates. In: International Joint Conference on Neural Networks (IJCNN 2014), Beijing, 2014; pp.82-92.

[96] Pham D T, Ghanbarzadeh A, Koc E, Otri S, Rahim S, Zaidi M. The bees algorithm-a novel tool for complex optimization problems. Technical Note, Manufacturing Engineering Centre, Cardiff University, UK. 2005. doi: 10.1016/B978-008045157-2/50081-X.

[97] Babu B G, Kannan M. Lightning bugs. Resonance, 2002; 7: 49-55.

[98] Memmah M, Lescourret F, Yao X, Lavigne C. Metaheuristics for agricultural land use optimization. A review. Agron. Sustain. Dev., 2015; 35: 975-998. 